\title{
Note on invariant properties of a quantum system placed into thermodynamic environment
}

\author{
(Published: Physica A 398 (2014) 65-75) \\ A. Y. Klimenko* \\ The University of Queensland, SoMME \\ QLD 4072, Australia
}

May 28, 2021

\begin{abstract}
The analysis conducted in this work indicates that interactions of a CP-violating (and CPT-preserving) quantum system with a thermodynamic environment can produce the impression of a CPT violation in the system. This conclusion is reasonably consistent with the results reported for decays of neutral K-mesons.
\end{abstract}

Keywords: qantum thermodynamics, CPT invariance

\section{Introduction}

The influence of a thermodynamic environment, commonly referred to as a thermodynamic bath, on quantum systems has been repeatedly discussed in publications. Zurek [1] introduced a theory explaining loss of coherence in a quantum system under the influence of the environments that have a large number of degrees of freedom. Goldstein et. al. 2, Popescu et. al. 3 and others demonstrated the property called canonical typicality: for most pure states of the environment, the quantum system behaves as if the environment was in the thermodynamic (i.e. maximally mixed) state. Linden et. al. [4] proved that under certain conditions the evolution of a quantum system placed into a bath leads to equilibration. These and other aspects of thermalisation have been reviewed by Yukalov [5], who stressed that, practically, any quantum system cannot be completely isolated and is subject to some influence from the environment. The existence of a degree of similarity between thermodynamic and pure-state quantum engines has been discussed by Abe [6]

*Email for communications: klimenko@mech.uq.edu.au 
The goal of the present work is to show that thermodynamic environment can affect apparent invariant properties of a quantum system, whose intrinsic behaviour involves a CP-violation. Here we refer to charge, parity and time symmetries conventionally denoted by $\mathrm{C}, \mathrm{P}$ and $\mathrm{T}$ in quantum mechanics [7. While the overwhelming majority of known quantum effects are $\mathrm{CP}$-compliant, the case of $\mathrm{CP}$ violation in decay of K-mesons (kaons) has been known and investigated for many decades 8 .

While the present work focuses on the effects induced by the environment (assuming conventional unitarity of quantum evolutions) the likelihood of spontaneous (intrinsic) violations of quantum mechanics, which can coexist with induced mechanisms and also be responsible for thermalisation, has been repeatedly discussed in publications [9, 10, 11. The possibility that these violations can affect decays of K-mesons has been raised by Ellis et. al. 12. Although the influence of spontaneous violations of quantum mechanics can not be excluded a priori and needs to be considered, such violations remain outside the scope of the present work. Here, we consider the influence of the environment within the framework of conventional quantum mechanics while relying on causality and proper choice of parameters to reproduce the thermodynamic direction of time.

\section{Quantum system in a radiation bath}

Consider a quantum system, which involves both particles and antiparticles and is placed into environment filled by radiation. The radiation is equilibrated by surroundings, which, of course, are made of matter prevalently present in the Universe. The system under consideration is a quantum system but is subject at least to some thermodynamic influence from the environment. Since antiparticles cannot interact weakly (i.e. without annihilations) with an environment formed by matter, these interactions are performed only through radiation, which is always generated by surrounding matter having non-zero temperatures. Note that we do not consider stronger interaction of quantum system with radiation through emission or adsorption. Only very weak interactions of the system and radiation that tend to impose quantum decoherence on the system [1, 5] are of interest, while radiation refers to any field that can be responsible for such interactions. The system placed in a radiation bath, which is equilibrated by surroundings, is schematically depicted in Figure 1. This scheme of interactions of the system and the environment through the radiation bath reflects the fact that surrounding matter needs to be removed from direct contact with the system that involves both particles and antiparticles. We conduct our analysis within the limits of quantum mechanics and take into account the thermodynamic direction of time through causality and choice of the interaction parameters.

The state of the radiation bath is characterised by its set of energy eigenstates $\mathbb{H}_{B}|\beta\rangle=E_{\beta}|\beta\rangle$. The dimension of this system is very large. In the same way, the state of the environment, which, as some publications [3] prefer to describe,

may involve the rest of the universe, is characterised by an even larger set of 


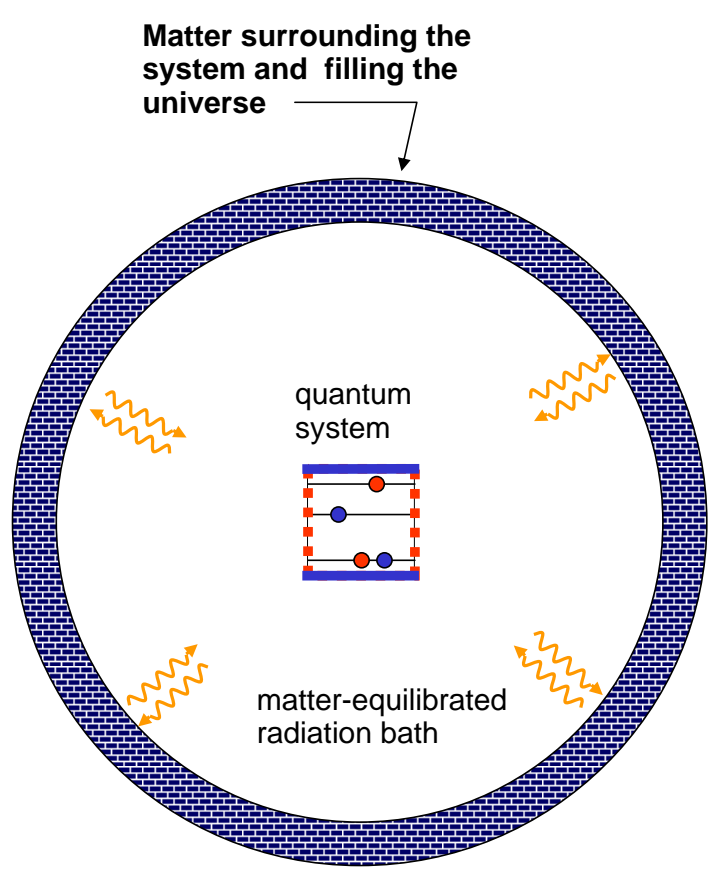

Figure 1: Quantum system placed into a radiation bath.

eigenstates $\mathbb{H}_{\Omega}|\Omega\rangle=E_{\Omega}|\Omega\rangle$. The state of the system can be described by a set of orthogonal ket states $|s\rangle$. Hence the overall state of the universal system involving the system, the bath and the environment is specified by vectors in the tensor product space $|s\rangle \otimes|\beta\rangle \otimes|\Omega\rangle$ (and the corresponding bra space $\langle s| \otimes$ $\langle\beta| \otimes\langle\Omega|)$. Our analysis is conducted under several assumptions, which can be summarised by:

1. the quantum system is small and connected to a much larger environment through the radiation bath;

2. the radiation bath can exercise some influence on the system;

3. the system has little effect on the bath, which is equilibrated by the environment and remains in a thermodynamic state.

Our use of the words "strong" and "weak" generally pertains to common understanding of these terms, which nevertheless does not exclude links with the strong and electroweak interactions of particle physics. Although the presence of the environment must always be kept in mind, our assumptions lead to autonomous consideration of the supersystem, which involves only the system 
and the radiation bath. The state of the supersystem is specified by the tensor product, which can be equivalently denoted by $|s\rangle \otimes|\beta\rangle=|s\rangle|\beta\rangle=|s \beta\rangle$. Note that we do not intend to demonstrate that the bath should be in its thermodynamic state but simply introduce this physical fact as a principal postulate of our analysis.

\subsection{The interaction Hamiltonian}

The overall Hamiltonian of the supersystem can be conventionally written in the form

$$
\mathbb{H}=\mathbb{H}_{S} \otimes \mathbb{I}_{B}+\mathbb{I}_{S} \otimes \mathbb{H}_{B}+\mathbb{H}_{S B}
$$

where $\mathbb{H}_{S}$ is Hamiltonian of the system, $\mathbb{H}_{B}$ is the Hamiltonian of the bath, $\mathbb{H}_{S B}$ is the system/bath interaction Hamiltonian and $\mathbb{I}$ represents the corresponding identity operators. Our assumptions, as detailed below, correspond to the following form of the bath and interaction Hamiltonians

$$
\left\langle\alpha\left|\mathbb{H}_{B}\right| \beta\right\rangle=E_{\beta} I_{\alpha \beta}, \quad\left\langle q \alpha\left|\mathbb{H}_{S B}\right| s \beta\right\rangle=h_{q s}^{(\beta)} I_{\alpha \beta}
$$

Here, $I_{\alpha \beta}$ is the identity matrix $I_{\alpha \beta}=0$ for $\alpha \neq \beta$ and $I_{\alpha \beta}=1$ for $\alpha=\beta$. The corresponding indices run over the same sets of states, that is $\{s\}=\{q\}$ and $\{\beta\}=\{\alpha\}$.

The Hamiltonian specified by (2) does not change the state of the bath but may alter the behaviour of the system. Indeed, the supersystem wave function $\Psi$ can be written in the form of the sum

$$
\mid \Psi(t)\}=\sum_{\beta} p_{\beta}^{1 / 2}[\beta]\left|\Psi_{\beta}(t)\right\rangle
$$

where

$$
\left|\Psi_{\beta}(t)\right\rangle=\left|\psi^{(\beta)}\right\rangle|\beta\rangle,\left|\psi^{(\beta)}\right\rangle=\sum_{s} c_{s \beta}(t)|s\rangle
$$

Dependence on time can be omitted for brevity when this does not cause confusion, for example $\mid \Psi\}=\mid \Psi(t)\}$. Here, the random phases $[\beta]$ indicate that $\mid \Psi\}$ is not a pure state but is a mixture of pure states $\left|\Psi_{\beta}\right\rangle$ with probabilities $p_{\beta}$. The notations involving the random phases and their links with the density matrix (operator)

$$
\mathbb{P}(t)=\mid \Psi\}\left\{\Psi\left|=\sum_{s, q, \beta} p_{\beta} c_{s \beta}(t) c_{q \beta}^{*}(t)\right| s \beta\right\rangle\langle q \beta|
$$

are explained in Appendix A. The shape of the density matrix $p$ depends on presence of $[\beta]$ in $[3]$, which forces $\beta=\alpha$ by $\left\langle[\alpha]^{*}[\beta]\right\rangle=I_{\alpha \beta}$ in $(5)$.

Hamiltonian (2) corresponds to our assumption 3 as well as to the action of quantum decoherence [1, 5] and canonical typicality [2, 3. This Hamiltonian ensures that not only $\mid \Psi\}$ but also every $\left|\Psi_{\beta}\right\rangle$ represents a solution of the Schrodinger equation for the supersystem

$$
i \frac{\partial}{\partial t}\left|\Psi_{\beta}(t)\right\rangle=\mathbb{H}\left|\Psi_{\beta}(t)\right\rangle
$$


For simplicity, the time $t$ is measured in Planck constants $\hbar$. If $\left|\Psi_{\beta}\right\rangle$ was not an autonomous solution, each $\left|\Psi_{\beta}\right\rangle$ would evolve into a superposition of pure states involving different $\left|\beta_{1}\right\rangle,\left|\beta_{2}\right\rangle, \ldots$ This would contradict our understanding [1, 2, 3, 4, 5, that interactions of the bath with the environment should decohere the bath eigenstates $\left|\beta_{1}\right\rangle,\left|\beta_{2}\right\rangle, \ldots$ and dissolve any superposition of these states into a mixture. The presence of a small system should not change the behaviour of the bath. The wave function of the bath is deemed to stay in thermal equilibrium specified by the mixed state

$$
\left.\mid \Psi_{B}\right\}=\sum_{\beta} p_{\beta}^{1 / 2} e^{-i E_{\beta} t}[\beta]|\beta\rangle, \quad p_{\beta} \sim \exp \left(\frac{-E_{\beta}}{k_{B} T}\right)
$$

Note that although the eigenstates of the bath are not affected by interactions with the system according to 2 , the eigenvalues $E_{\beta}$ can in principle be altered by these interactions. This alteration, however, is practically insignificant and neglected here - consider that the bath is more affected by the environment than by the system.

\subsection{Reduced density matrix and wave function of the sys- tem}

The behaviour of the quantum system is conventionally characterised by the reduced density operator

$$
\mathbb{P} S(t)=\sum_{\beta}\langle\beta|\mathbb{P}| \beta\rangle=\sum_{s, q}\left(\rho_{S}\right)_{s q}|s\rangle\langle q|, \quad\left(\rho_{S}\right)_{s q}=\sum_{\beta} p_{\beta} c_{s \beta}(t) c_{q \beta}^{*}(t),
$$

which is obtained from the density p specified in (5) by tracing the bath states out. One can easily see that the mixed state of the system specified by the wave function

$$
\left.\mid \Psi_{S}(t)\right\}=\sum_{\beta} p_{\beta}^{1 / 2}[\beta]\left|\psi^{(\beta)}\right\rangle,
$$

where $\left|\psi^{(\beta)}\right\rangle$, given by $(4)$, is equivalent to the density operator specified by (8). That is $\left.\mathbb{p}_{S}=\mid \Psi_{S}\right\}\left\{\Psi_{S} \mid\right.$, where the random phase rule $\left\langle[\alpha]^{*}[\beta]\right\rangle=I_{\alpha \beta}$ applies. This equivalence implies that, for any system observable $Q_{S}$, the values found from the density matrix and from the wave function are indistinguishable to the observer

$$
Q_{S}=\operatorname{tr}\left(\mathbb{P}_{S} \mathbb{Q}_{S}\right)=\left\{\Psi_{S}\left|\mathbb{Q}_{S}\right| \Psi_{S}\right\}=\sum_{\beta} p_{\beta} Q_{S}^{(\beta)}, \quad Q_{S}^{(\beta)}=\left\langle\psi^{(\beta)}\left|\mathbb{Q}_{S}\right| \psi^{(\beta)}\right\rangle
$$

and so are the mixed states specified by (8) and $(9)$. Hence, we can use $\left.\mid \Psi_{S}(t)\right\}$, which is called here the reduced wave function, as a convenient tool for investigating of the state of the system. Note that in this case the reduced wave function $\left.\mid \Psi_{S}(t)\right\}$ specified by $(9)$ can be obtained from the wave function of the 
supersystem $\mid \Psi(t)\}$ specified by (3) through the following procedure: multiplying $\mid \Psi\}$ by the bra $\langle\alpha|$, summing over all $\alpha$

$$
\left.\mid \Psi_{S}(t)\right\}=\sum_{\alpha}\langle\alpha|| \Psi\}=\sum_{\alpha, \beta}\langle\alpha \mid \beta\rangle p_{\beta}^{1 / 2}[\beta]\left|\psi^{(\beta)}\right\rangle
$$

and noting orthonormality $\langle\alpha \mid \beta\rangle=I_{\alpha \beta}$. The double " |" indicates incompleteness of the inner product since the ket spans over a wider space $\mid \Psi\} \sim|s\rangle|\beta\rangle$ than the bra $\langle\alpha|$.

The system wave functions $\left|\Psi_{S}^{(\beta)}\right\rangle$, which are similar to $\left|\psi^{(\beta)}(t)\right\rangle$ but have the effect of the bath energy eigenstates $E_{\beta}$ factored out, are introduced by

$$
\left|\psi^{(\beta)}(t)\right\rangle=e^{-i E_{\beta} t}\left|\Psi_{S}^{(\beta)}(t)\right\rangle,
$$

that is

$$
\left|\Psi_{S}^{(\beta)}(t)\right\rangle=\sum_{s} C_{s}^{(\beta)}(t)|s\rangle, \quad C_{s}^{(\beta)}=e^{i E_{\beta} t} c_{s \beta}
$$

The wave functions $\left|\Psi_{S}^{(\beta)}\right\rangle$ can now be considered independently from each other. Note that the expression for the reduced wave function remains the same

$$
\left.\mid \Psi_{S}(t)\right\}=\sum_{\beta} p_{\beta}^{1 / 2}\left[\beta^{\circ}\right]\left|\Psi_{S}^{(\beta)}(t)\right\rangle, \quad\left[\beta^{\circ}\right]=e^{-i E_{\beta} t}[\beta]
$$

since, as discussed in the Appendix, the random phases $\left[\beta^{\circ}\right]$ are functionally equivalent to $[\beta]$. Each $\left|\Psi_{S}^{(\beta)}\right\rangle$ represents an independent solution of the reduced Schrodinger equation

$$
i \frac{\partial}{\partial t}\left|\Psi_{S}^{(\beta)}(t)\right\rangle=\mathbb{H}^{(\beta)}\left|\Psi_{S}^{(\beta)}(t)\right\rangle
$$

with the $\beta$-dependent effective system Hamiltonian $\mathbb{H}^{(\beta)}$ specified by

$$
\begin{aligned}
\mathbb{H}^{(\beta)} & =\langle\beta|\mathbb{H}| \beta\rangle-E_{\beta} \mathbb{I}_{S}=\mathbb{H}_{S}+\mathbb{h}^{(\beta)} \\
\mathbb{h}^{(\beta)} & =\left\langle\beta\left|\mathbb{H}_{S B}\right| \beta\right\rangle=\sum_{s, q} h_{s q}^{(\beta)}|s\rangle\langle q|
\end{aligned}
$$

Equation 14 can be obtained by multiplying $(6)$ by $\langle\beta|[\beta]^{*}$ and factorising $e^{i\left(\mathbb{I}_{S} \otimes \mathbb{H}_{B}\right) t}$ from the rest of the solution. Here, we take into account that the operators $\mathbb{H}$ and $\mathbb{I}_{S} \otimes \mathbb{H}_{B}$ commute $\left[\mathbb{H}, \mathbb{I}_{S} \otimes \mathbb{H}_{B}\right]=0$ as long as $\mathbb{H}_{B}$ and $\mathbb{H}_{S B}$ are defined by (2). Note that the operators $\mathbb{H}$ and $\mathbb{H}_{S} \otimes \mathbb{I}_{B}$ are not necessarily commutative due to the possibility of $\left[\mathbb{H}_{S}, \mathbb{h}^{(\beta)}\right] \neq 0$. 


\subsection{On unitarity of quantum models and decoherence}

From the perspective of the canonical typicality, the state of the bath - maximally mixed or a typical pure state - should have little effect on the properties of the system characterised by reduced quantities. While being important, this property does not change our treatment since the bath is physically in thermodynamic equilibrium with the environment. When considered from the perspective of interactions of the small system with the large bath, the microcanonical maximally mixed state of the bath (i.e. state with maximal entropy and fixed total energy) is essentially the same as the canonical equilibrium state of the bath specified by (7). The main effect of the bath on the state of the system is in inducing decoherence of the eigenstates of the system, which is not the same as decoherence of the wave functions multiplied by different random phases $[\beta]$ in (3) and (9). Assuming that the states $|s\rangle$ are energy eigenstates of the system in the absence of interference from the bath (this is assumed only in this subsection), the decoherence of these eigenstates results in

$$
\left(\rho_{S}\right)_{s q} \approx 0 \text { for } s \neq q
$$

that is the reduced density matrix becomes diagonal. This property is not synonymous with the decoherence of $[\beta]\left|\Psi_{S}^{(\beta)}\right\rangle$.

The theory of quantum decoherence induced by interactions with a bath or environment has been introduced by Zurek [1. This theory is based on assuming that the interaction term is diagonal, that is

$$
h_{s q}^{(\beta)}=h_{s}^{(\beta)} I_{s q}
$$

implying that operators $\mathbb{H}_{S}$ and $\mathbb{h}^{(\beta)}$ commute

$$
\left[\mathbb{H}_{S}, \mathbb{h}^{(\beta)}\right]=0
$$

Note that (18) and (19) are assumptions of the conventional decoherence theory [1] and not of the present work. The solution of equation (14) is then easily obtainable

$$
\left|\Psi_{S}^{(\beta)}(t)\right\rangle=\exp \left(-i\left(E_{s}+h_{s}^{(\beta)}\right) t\right)
$$

resulting in

$$
\left(\rho_{S}\right)_{s q}=A_{s q} \exp \left(-i\left(E_{s}-E_{q}\right) t\right), \quad A_{s q}=\sum_{\beta} p_{\beta} \exp \left(-i\left(h_{s}^{(\beta)}-h_{q}^{(\beta)}\right) t\right)
$$

As a sum of a large number of uncorrelated values, $A_{s q}$ should be small on average assuming that the number of probable states indexed by $\beta$ is large and, of course, that $q \neq s[1$.

While decoherence is an essentially non-unitary (and irreversible) process increasing the entropy, the description of induced decoherence given above is based on placing the system into a bath to form a supersystem of a much 
larger dimension than that of the system. However, even a very large supersystem is still described by its Schrodinger equation (6), which is unitary and, consequently, time reversible. Is this reversible description consistent with the irreversible reality? This question can be traced back to historic discussions between Boltzmann, Loschmidt and Zermelo. It should be noted first that both canonical typicality and induced decoherences deal with average, typical properties that may, without contradicting the formulation of these statements, be subject to some rare exceptions. For example, $A_{s q}(t)$ is a quasiperiodic function of time in (21) and, eventually, should recur to the proximity of its initial value $A_{s q}(0)$, but, in large supersystems, these recurrences occur after extremely long times and would be impossible to observe in practice. A very small correction, uncertainty or external interference added to the model would be sufficient to remove these recurrences. While, as remarked in the Introduction, spontaneous violations of unitarity are also likely to play a role in decoherence and thermalisation, the present work focuses on the induced mechanisms of decoherence. Unitary evolution of a sufficiently large dimension combined with the causality principle can provide a very good approximation for irreversible evolutions observed in reality.

\subsection{Decay kinetics}

This section introduces an influence of the environment into the WeisskopfWigner approximation [13. Since the 1930s, this approximation is conventionally used for characterisation of decaying states. The Weisskopf-Wigner approximation is derived from the Schrodinger equation for specific initial conditions and under assumptions of relatively weak interactions of the few initial with many final states. Later publications [14] demonstrated the rigorous asymptotic character of this approximation.

Among the system states $|s\rangle$, we distinguish two groups: the initial state or states $|k\rangle(\langle j|$ is used when an alternative index is needed for the bra-space), and many possible final states, which are indexed by $|f\rangle$, that is $\{s\}=\{k, f\}$ and $\{k\}=\{j\}$. The system is initially placed into a pure state that spans over one or several $k$-states but then decays into one or several of the $f$-states. The system Hamiltonian is given by $\mathbb{H}_{S}=\mathbb{H}_{0}+\mathbb{H}_{1}$, where the smaller component $\mathbb{H}_{1}$ is responsible for relatively weak interactions of the initial and final states. The states $|s\rangle$, involving the initial $|k\rangle$ and final $|f\rangle$ states, are eigenstates $\mathbb{H}_{0}|s\rangle=E_{s}|s\rangle$ of the undisturbed Hamiltonian $\mathbb{H}_{0}$ (i.e. strong eigenstates) but not necessarily the eigenstates of $\mathbb{H}_{S}$. If there are several initial states (for example a particle and its antiparticle), then these states are assumed to possess the same energy $E_{k}=E_{0}$ for all $k$. After tracing out the state of the environment from the Hamiltonian, we rewrite (1) as

$$
\mathbb{H}^{(\beta)}=\langle\beta|\mathbb{H}| \beta\rangle-E_{\beta} \mathbb{I}_{S}=\mathbb{H}_{0}+\mathbb{H}_{2}^{(\beta)}, \quad \mathbb{H}_{2}^{(\beta)} \equiv \mathbb{H}_{1}+\mathbb{h}^{(\beta)}
$$


The matrix $\Lambda_{k j}^{(\beta)}$, which specifies the evolution of the $k$-states

$$
i \frac{d}{d t}\left|\Psi_{k}^{(\beta)}\right\rangle=\sum_{j} \Lambda_{k j}^{(\beta)}\left|\Psi_{j}^{(\beta)}\right\rangle
$$

is obtained by applying the Weisskopf-Wigner approximation [13] to equation (14)

$$
\Lambda_{j k}^{(\beta)}=\left\langle j\left|\mathbb{H}^{(\beta)}\right| k\right\rangle+\lambda_{j k}^{(\beta)}, \quad \lambda_{j k}^{(\beta)} \equiv \sum_{f} \frac{\left\langle j\left|\mathbb{H}_{2}^{(\beta)}\right| f\right\rangle\left\langle f\left|\mathbb{H}_{2}^{(\beta)}\right| k\right\rangle}{E_{0}-E_{f}+i \varepsilon}
$$

where $\varepsilon \rightarrow 0$ and the sign of $\varepsilon$ is selected to produce decaying exponents as required by causality. The matrix $\Lambda_{j k}^{(\beta)}$ is generally not Hermitian, while Her$\operatorname{mitian} M_{j k}^{(\beta)}$ and $\Gamma_{j k}^{(\beta)}$ in

$$
\Lambda_{j k}^{(\beta)}=M_{j k}^{(\beta)}-\frac{i}{2} \Gamma_{j k}^{(\beta)}
$$

represent the energy-mass matrix and the decay matrix correspondingly. The imaginary component of the sum $\lambda_{j k}^{(\beta)}$, which is associated with the term $\Gamma_{j k}^{(\beta)}$, appears as the contribution of so-called "on-shelf" states $\hat{f}$ where $E_{0}=E_{\hat{f}}$. The states $\check{f}$, representing the remaining or "off-shelf" states in $\{f\}=\{\hat{f}, \check{f}\}$, contribute only to $M_{j k}^{(\beta)}$. Since the set of final states is large and, possibly, continuous, the sum in 24) is understood and evaluated as the corresponding integral.

\subsection{The interaction term and the eigenstates}

According to Zurek's theory [1], the terms responsible for decoherence act primarily along the eigenstates of the system - see the discussion around equation (19). Applying the same strategy to the present case encounters difficulties for the on-shelf states that are discussed below. These difficulties indicate that (19) is excessively restrictive and not suitable for the present analysis.

If we restrict our attention only to the strongest interactions specified by the undisturbed Hamiltonian $\mathbb{H}_{0}$ then at the leading order, this implies that $\mathbb{h}^{(\beta)}$ is diagonal

$$
\left\langle q\left|\mathbb{h}^{(\beta)}\right| s\right\rangle \approx h_{s}^{(\beta)} I_{q s}
$$

This expression is responsible for interference of the bath with the initial $|k\rangle$ states and final $|f\rangle$ states autonomously without any transition from $|k\rangle$ to $|f\rangle$, while we are interested in interference of the bath with the process of the transition.

One might try to correct the set of eigenstates $\{|s\rangle\}=\{|k\rangle,|f\rangle\}$ to account for weak interactions specified by $\mathbb{H}_{1}$. At the leading order, the corrected eigenstates $\left|s^{\prime}\right\rangle$ are given by the standard quantum perturbation theory [15] yielding

$$
\left|s^{\prime}\right\rangle=|s\rangle+\sum_{q \neq s} \frac{\left\langle q\left|\mathbb{H}_{1}\right| s\right\rangle}{E_{s}-E_{q}}|q\rangle+\ldots
$$


Assuming that, according to $(19)$, the decohering term is aligned with the corrected eigenstates $\left|s^{\prime}\right\rangle$ we obtain

$\mathbb{h}^{(\beta)}=\sum_{s} h_{s}^{(\beta)}\left|s^{\prime}\right\rangle\left\langle s^{\prime}\left|=\sum_{s} h_{s}^{(\beta)}\right| s\right\rangle\left\langle s\left|+\sum_{s, q \neq s}\left(h_{s}^{(\beta)}-h_{q}^{(\beta)}\right) \frac{\left\langle q\left|\mathbb{H}_{1}\right| s\right\rangle}{E_{s}-E_{q}}\right| q\right\rangle\langle s|+\ldots$

Substitution of (28) and (22) into (24) produces integrals (represented by sums over $f$ in the equations) which diverge as $\left(E_{0}-E_{f}\right)^{-2}$ in vicinities of the on-shelf states $\hat{f}$, where $E_{0}=E_{f}$. This shows that bath interference with decay is mostly contributed to by the vicinities of the on-shelf states $\hat{f}$ and, at the same time, indicates that our approximations and assumptions need to be reevaluated in these vicinities. Since the exact sub-atomic mechanisms of decoherence remain unknown, our consideration is necessarily based on qualitative analysis of the system/bath interaction term.

Since the on-shelf states $\hat{f}$ are degenerate due to $E_{0}=E_{f}$, the eigenstates experience large and rapid changes in vicinities of these states, induced by higher order terms in the Hamiltonian [15] (the degeneracy of the initial states $E_{k}=E_{0}$ is uniformly present in all transitions and does not cause rapid adjustments). Although the interference terms are likely to be affected when $f \rightarrow \hat{f}$, these terms can not be assumed to be fully aligned with the rapidly changing eigenstates in vicinity of the on-shelf states. Hence, the interference term is no longer compliant with (19), when the on-shelf states are involved. This implies interactions between different energy modes. While the decoherence theory [1] presumes decoherence at short times without energy exchange, decays are more affected by interference with the bath and involves some redistribution of energy in vicinity of the on-shelf states.

\section{Invariant properties}

We note that the overwhelming majority of quantum effects that we know of are CP-invariant. Only very few exceptions have been found that contradict this rule. We assume that the system under consideration may be one of these exceptions, i.e. CP-violating and CPT-invariant. Note that here we refer only to the intrinsic properties of the system and not to the interaction of the system and the environment.

For interactions of the system and the thermodynamic environment, we assume $\mathrm{CP}$ invariance and, possibly, $\mathrm{T}$ violation. These assumptions need further comments. The current understanding of the influence of the environment on quantum systems [1, 2, 3, 4, 5] does not have any provisions for discriminating between its effects on particles and antiparticles; hence interactions of the system and the bath are expected to be CP-invariant. As there is no proof or assertion that these interactions must be T-invariant, we should allow for the possibility of $\mathrm{T}$ violations in environmental interactions.

The last point needs a more detailed discussion. Thermodynamic interactions taking place far from equilibrium are time directional and irreversible. For 
example, decoherence always occurs only forward and not backward in time. A number of theories [9, 10, 11, 16, relate macroscopic irreversibility to intrinsic microscopic processes at quantum level. These processes are expected to violate both quantum mechanics and microscopic time symmetry while having very small magnitudes that make any direct detection of the time-generating processes very difficult. In general, these time-generating violations of unitarity should violate either CPT or CP symmetry, giving rise to two possible alternative thermodynamics of interactions of matter and antimatter [17. In this work, however, we do not consider violations of quantum mechanics and focus our attention on the influence of the environment but note that the a priori presumption of time symmetry in interactions of a quantum system and its thermodynamic environment (which effectively encompasses the rest of the universe) would not be justified.

One may notice that our interpretation of the system's interactions with the universe - CP-invariance combined with possible T-violation - may constitute a CPT-violation. In general, CPT invariance is a theorem in quantum field theory but its validity in the context of statistical or general physics would be only a hypothesis. While some published opinions (see, for example, Penrose [16]) favour the view that the processes enacting the second law of thermodynamics are CPT-violating and that CPT invariance is not a property of the entire universe, we give a different interpretation for the case considered here. If it exists, CPT violation in thermodynamic interactions with the environment is only an apparent feature since the operation of charge conjugation is not rigorous in our setup. Indeed, under charge conjugation, we correctly swap particle and antiparticles in the system and correctly leave the radiation bath unchanged but we do not and cannot appropriately adjust the environment (i.e. change matter populating the universe into antimatter). Thus our operator $\mathrm{C}$ is, strictly speaking, incomplete, creating opportunities for apparent CPT violations when interactions with the environment are of some influence. According to the line of thinking adopted in quantum thermodynamics 1, 2, 3, 4, 5, interactions with the environment are deemed to be unavoidable.

Approximation (24) is conventionally used to analyse decay of neutral kaons, $K^{\circ}$ and $\bar{K}^{\circ}[8$. Here, we investigate the CPT-compliance of the decay, which requires that the term

$$
\Delta \Lambda^{(\beta)}=\Lambda_{K K}^{(\beta)}-\Lambda_{\bar{K} \bar{K}}^{(\beta)}
$$

is nullified [8]. Unlike the running indices $k$ and $j$, the subscripts $K$ and $\bar{K}$ denote the fixed states corresponding to the kaon and antikaon.

\subsection{Invariance of the system/bath interactions}

The property of radiation-induced interactions being CP-invariant and $\mathrm{T}$-asymmetric can be expressed in terms of the interaction Hamiltonian

$$
\begin{gathered}
\left\langle q \alpha\left|\mathbb{H}_{S B}\right| s \beta\right\rangle=\left\langle\overline{q \alpha}\left|\mathbb{H}_{S B}\right| \overline{s \beta}\right\rangle \Rightarrow h_{q s}^{(\beta)}=h_{\bar{q} \bar{s}}^{(\beta)} \\
\Delta h_{q s}^{(\beta)}=\left\langle q \beta\left|\mathbb{H}_{S B}\right| s \beta\right\rangle-\left\langle s \beta\left|\mathbb{H}_{S B}\right| q \beta\right\rangle \neq 0
\end{gathered}
$$


where the overbars denote anti-states. The property $\beta=\bar{\beta}$ is taken into account for radiation (i.e. matter and antimatter interact with radiation in the same way). Note that $\Delta h_{q s}^{(\beta)}$ is imaginary when interactions with the environment are unitary and $\mathbb{h}^{(\beta)}$ is Hermitian (if $\mathbb{h}^{(\beta)}$ were the term responsible for violations of unitarity in thermodynamic interactions, this would correspond to real $\left.\Delta h_{q s}^{(\beta)}\right)$. As previously noted, equations (30) and (31) imply an apparent CPT violation:

$$
\left\langle q \beta\left|\mathbb{H}_{S B}\right| s \beta\right\rangle \neq\left\langle\overline{s \beta}\left|\mathbb{H}_{S B}\right| \overline{q \beta}\right\rangle
$$

\subsection{The case of a CP-invariant system}

Most quantum systems possess CP symmetry and relatively few $\mathrm{CP}$ violations are known in quantum mechanics. When the system Hamiltonian $\mathbb{H}_{S}$ is intrinsically CP-invariant, we obtain

$$
\begin{aligned}
H_{k f} & =\left\langle k\left|\mathbb{H}_{S}\right| f\right\rangle=\left\langle\bar{k}\left|\mathbb{H}_{S}\right| \bar{f}\right\rangle=H_{\bar{k} \bar{f}} \\
\Delta \Lambda^{(\beta)} & \equiv \Lambda_{K K}^{(\beta)}-\Lambda_{\bar{K} \bar{K}}^{(\beta)}=0
\end{aligned}
$$

Here we use equations (30), (24), (33) to obtain (34) and conclude that the system appears to the observer as being CPT-compliant. Here, we refer only to opinion of the observer, who uses $\Delta \Lambda^{(\beta)}$ to test the CPT-invariance; in general, the system may or may not be CPT-invariant under these conditions. We also note that, since the Hamiltonians $\mathbb{H}_{0}$ and $\mathbb{H}_{2}^{(\beta)}$ are CP-preserving, there is no

$\mathrm{T}$ violation in this system apparent to the observer $\Lambda_{K \bar{K}}^{(\beta)}=\Lambda_{\bar{K} K}^{(\beta)}$, although, once again, the system's interactions with the environment may in fact be $\mathrm{T}$ violating.

\subsection{The case of a CP-violating and CPT-invariant system}

The system Hamiltonian $\mathbb{H}_{S}$ is CPT-invariant provided that

$$
H_{k f}=\left\langle k\left|\mathbb{H}_{S}\right| f\right\rangle=\left\langle\bar{f}\left|\mathbb{H}_{S}\right| \bar{k}\right\rangle=H_{\bar{f} \bar{k}}
$$

We use equations (30, $(24)$ and (35) and note that the first term in (24) does not contribute to $\Delta \Lambda^{(\beta)}$ so that

$$
\begin{gathered}
\Delta \Lambda^{(\beta)}=\lambda_{K K}^{(\beta)}-\lambda_{\bar{K} \bar{K}}^{(\beta)} \\
=\sum_{f} \frac{\left(H_{K f}^{\prime}+h_{K f}^{(\beta)}\right)\left(H_{f K}^{\prime}+h_{f K}^{(\beta)}\right)-\left(H_{\bar{K} \bar{f}}^{\prime}+h_{\bar{K} \bar{f}}^{(\beta)}\right)\left(H_{\bar{f} \bar{K}}^{\prime}+h_{\bar{f} \bar{K}}^{(\beta)}\right)}{E_{0}-E_{f}+i \varepsilon} \\
=\sum_{f} \frac{\left(H_{K f}^{\prime}+h_{K f}^{(\beta)}\right)\left(H_{f K}^{\prime}+h_{f K}^{(\beta)}\right)-\left(H_{f K}^{\prime}+h_{K f}^{(\beta)}\right)\left(H_{K f}^{\prime}+h_{f K}^{(\beta)}\right)}{E_{0}-E_{f}+i \varepsilon} \\
=-\sum_{f} \frac{\Delta H_{K f}^{\prime}}{E_{0}-E_{f}+i \varepsilon} \Delta h_{K f}^{(\beta)}
\end{gathered}
$$


where we denote $H_{k f}^{\prime} \equiv\left\langle k\left|\mathbb{H}_{1}\right| f\right\rangle, \quad \Delta H_{k f}^{\prime}=H_{k f}^{\prime}-H_{f k}^{\prime}$. As such, thermodynamic interactions with the environment through radiation may appear in $\mathrm{CP}$-violating (but CPT preserving) systems as an apparent $\mathrm{CPT}$ violation. Absence of the time symmetry $\Delta H_{K f}^{\prime} \neq 0$ and $\Delta h_{K f}^{(\beta)} \neq 0$ is essential for this effect. Note that the product $\Delta H_{K f}^{\prime} \Delta h_{K f}^{(\beta)}$ is always real, since $\Delta H_{K f}^{\prime}$ and $\Delta h_{K f}^{(\beta)}$ are both imaginary. As discussed in Section 2.4, the major contribution to the sum 36 takes place in the vicinities of the on-shelf states $\hat{f}$ where $h_{s q}^{(\beta)}$ is not restricted to the diagonal $I_{s q}$ and $\Delta h_{s q}^{(\beta)}$ is non-zero.

\section{Tests of CPT invariance in kaon decays}

Decay of neutral kaons is one of very few known cases of CP-violation in quantum systems (it was the only known case of CP-violation for several decades and is widely covered in the literature see, for example, [8, 18]). The CP violation takes the form $\Lambda_{K \bar{K}} \neq \Lambda_{\bar{K} K}$, were we omit the superscript $\beta$ since the CP violation is induced by $\Delta H_{k f}^{\prime} \neq 0$ in 24 and does not need any interaction with the environment (i.e. we can put $h_{k f}^{(\beta)}=0$ ). This $\mathrm{CP}$ violation is conventionally understood as preserving $\mathrm{CPT}$ and violating $\mathrm{T}$ invariance.

The CPT invariance of kaon decays has also been repeatedly tested [8, 18]. The results of these tests are summarily presented in a recent review of particle physics [18] and are shown in Figure 2. These results neither constitute an unambiguous CPT violation nor assert the CPT invariance, although there is a noticeable CPT-violating bias in the results. The magnitude of the apparent $\mathrm{CPT}$ violation is only 10 times smaller than that of the established $\mathrm{CP}$-violation in this system but the relative scattering of the results is much larger in CPT than in $\mathrm{CP}$ tests which prevents any conclusive statements on CPT invariance.

Our explanation of the bias is that the apparent CPT violation in kaon decays is induced by thermodynamic interactions with the environment (and does not contradict to a rigorous interpretation of CPT invariance). This violation can be traced to the fact that, in our experiments, we cannot possibly perform charge conjugation of the environment. As discussed below, this explanation seems to be consistent with the results presented in Figure 2.

The apparent CPT violation in (36) is caused by interference of the environment with quantum interactions of the initial and the on-shelf final states of the system. Hence, the contribution to the violation from the sum in $(36)$ is dominated by the vicinities of the on-shelf states $\hat{f}$. Since the product $\Delta H_{K f}^{\prime} \Delta h_{K f}^{(\beta)}$ is always real, this product contributes to both the real $\Delta M^{(\beta)} \equiv M_{K K}^{(\beta)}-M_{\bar{K} \bar{K}}^{(\beta)}$ and imaginary $\Delta \Gamma^{(\beta)} \equiv \Gamma_{K K}^{(\beta)}-\Gamma_{\bar{K} \bar{K}}^{(\beta)}$ components of $\Delta \Lambda^{(\beta)}$. This implies that, if only the realisations $\beta$ that possess the property $\Delta \Gamma^{(\beta)} \approx 0$ (due to $\Delta h_{K f}^{(\beta)}$ being small in the vicinity of $\hat{f}$ ) are selected, then the corresponding energymass term should also vanish $\Delta M^{(\beta)} \approx 0$. This expectation is consistent with the experimental results presented in Figure 2. Another feature of equation 36 is its linearity with respect to the fluctuating term $\Delta h_{K f}^{(\beta)}$, which does not 


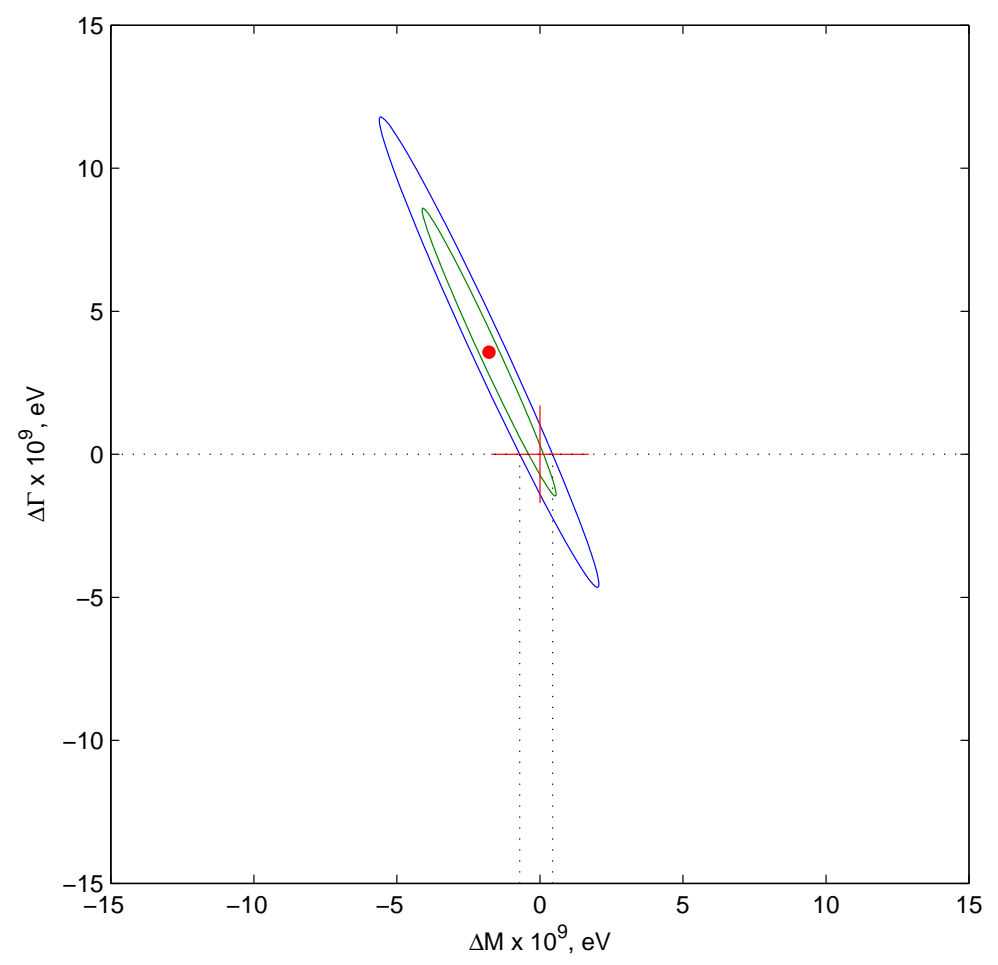

Figure 2: Test of CPT invariance in kaon decays [18. The two contours show the $95 \%$ and $65 \%$ confidence levels. The solid dot corresponds to the mean value. The cross indicates the coordinate origin - the point where no CPT violation occurs. The vertical dashed lines show the $95 \%$ confidence region for $\Delta M$ in realisations conditioned on $\Delta \Gamma=0$.

contradict the results shown in Figure 2 that seem to indicate the existence of a linear correlation or connection between $\Delta \Gamma^{(\beta)}$ and $\Delta M^{(\beta)}$. Fluctuations of large magnitudes (i.e. of the same order as mean values) are also consistent with the thermodynamic origin of the fluctuations, since microscopic systems placed in a thermodynamic environment are subject to fluctuations of magnitude comparable to the corresponding mean values.

\section{Conclusions}

The perspective suggested in this work is based on assumption that the thermodynamic influence of the environment is omnipresent in the real world; insulating experimental systems only weakens this influence but cannot exclude it completely. Although decays have stronger coupling with thermodynamic envi- 
ronment than quantum steady states, the direct influence of the environment on invariant properties may be difficult to notice in systems that are CP-invariant - the overwhelming majority of quantum systems belong to this group. In the case of intrinsic $\mathrm{CP}$ violations, interference of the environment is likely to produce the impression of a CPT violation: the decay of neutral kaons seems to support this view. This violation is only apparent and does not contradict strict interpretations of the CPT symmetry, which is fundamental in particle physics.

\section{Acknowledgments}

The author's work is supported by the Australian Research Council.

\section{APPENDIX: The random phase notation}

This appendix explains the system of notation that is based on random phases and used in this paper. These notations can be useful in distinguishing pure and mixed states of quantum mechanics, especially in cases where dealing with wave functions seems to be more convenient than using density matrices. We stress that the random phases serve only to provide notional convenience and do not represent a physical explanation or a theory. Let

$$
[\beta]=\exp \left(-i \theta_{\beta}\right), \quad[\beta]^{*}=\exp \left(+i \theta_{\beta}\right),
$$

where $\theta_{\beta}$ is a random angle uniformly distributed between 0 and $2 \pi$. When a wave function is multiplied by a random phase, evaluation of the density matrix involves averaging over the random phases, which is obviously compliant with the following rule

$$
\left\langle[\alpha]^{*}[\beta]\right\rangle=\left\langle\exp \left(-i\left(\theta_{\beta}-\theta_{\alpha}\right)\right)\right\rangle=I_{\alpha \beta}
$$

where $I_{\alpha \beta}$ is the identity matrix:

$$
I_{\alpha \beta}= \begin{cases}1 & \alpha=\beta \\ 0 & \alpha \neq \beta\end{cases}
$$

Note that the product of two random phases $\left[\beta_{3}\right]=\left[\beta_{1}\right]\left[\beta_{2}\right]$ is also a random phase that is different from both $\left[\beta_{1}\right]$ and $\left[\beta_{2}\right]$ since $\left\langle\left[\beta_{3}\right]^{*}\left[\beta_{1}\right]\right\rangle=0$ and $\left\langle\left[\beta_{3}\right]^{*}\left[\beta_{2}\right]\right\rangle=0$. Multiplication of a random phase by a fixed phase is still a random phase, although not an independent one: $\left[\beta^{\circ}\right]=e^{-i \phi}[\beta]$ and $\left\langle\left[\beta^{\circ}\right]^{*}[\beta]\right\rangle=e^{i \phi}$.

Consider the following examples. The first expression

$$
\left|\psi_{1}\right\rangle=\sum_{\beta} c_{\beta}|\beta\rangle
$$


represents a pure state, which is a superposition of pure states $|\beta\rangle$. The second expression

$$
\left.\mid \psi_{2}\right\}=\sum_{\beta} c_{\beta}[\beta]|\beta\rangle
$$

represents a mixed state, which is a mixture of the pure states $|\beta\rangle$. Let us evaluate the corresponding density operators to see the difference

$$
\begin{gathered}
\mathbb{P}_{1}=\left|\psi_{1}\right\rangle\left\langle\psi_{1}\left|=\sum_{\alpha, \beta} c_{\alpha}^{*} c_{\beta}\right| \beta\right\rangle\langle\alpha| \\
\left.\mathbb{P}_{2}=\mid \psi_{2}\right\}\left\{\psi_{2}|=\sum_{\alpha, \beta} c_{\alpha}^{*} c_{\beta} \underbrace{\left\langle[\alpha]^{*}[\beta]\right\rangle}_{=I_{\alpha \beta}}| \beta\right\rangle\left\langle\alpha\left|=\sum_{\beta} c_{\beta} c_{\beta}^{*}\right| \beta\right\rangle\langle\beta|
\end{gathered}
$$

where rule (38) is applied. The second density operator corresponds to a mixed state.

In a mixed state, superposition and mixing can be performed over different indices. For example, the expression

$$
\mid \psi\}=\sum_{s, \beta} c_{s \beta}[\beta]|s\rangle
$$

represents the pure states

$$
\left|\psi_{\beta}\right\rangle=\sum_{s} \frac{c_{s \beta}}{p_{\beta}^{1 / 2}}|s\rangle, \quad \beta=1,2, \ldots
$$

that are mixed with the probabilities $p_{\beta}=\Sigma_{s} c_{s \beta}^{*} c_{s \beta}$.

Generally, the random phase notation is equivalent to notations using the density matrices, although in some cases the random phases can distinguish different mixed states that have identical density matrices. A good example, which has been mentioned in many publications [16], can be expressed in terms of the random phases by the following expressions

$$
\begin{aligned}
\left|\psi_{+1}\right\rangle & =\frac{|\uparrow\rangle+|\downarrow\rangle}{\sqrt{2}}, \quad\left|\psi_{-1}\right\rangle=\frac{|\uparrow\rangle-|\downarrow\rangle}{\sqrt{2}} \\
\left.\mid \psi_{2}\right\} & \left.=\frac{[\alpha]|\uparrow\rangle+[\beta]|\downarrow\rangle}{\sqrt{2}}, \quad \mid \psi_{3}\right\}=\frac{[\alpha]\left|\psi_{+1}\right\rangle+[\beta]\left|\psi_{-1}\right\rangle}{\sqrt{2}}
\end{aligned}
$$

The first two expressions specify $\left|\psi_{+1}\right\rangle$ and $\left|\psi_{-1}\right\rangle$ as pure states that are different superpositions of two other pure states $|\uparrow\rangle$ and $|\downarrow\rangle$ representing spin. The last two expressions indicate that $\left.\mid \psi_{2}\right\}$ is a mixture of two pure states $|\uparrow\rangle$ and $|\downarrow\rangle$ with equal probability and that $\left.\mid \psi_{3}\right\}$ is a mixture of two other pure states $\left|\psi_{+1}\right\rangle$ and $\left|\psi_{-1}\right\rangle$. Note that $\left.\mid \psi_{3}\right\}$ is different from $\left.\mid \psi_{2}\right\}$ and this difference is reflected by the random phase notation. The density operators corresponding to these cases are evaluated according to (43) and, in matrix form, are given by

$$
\begin{gathered}
\mathbb{P}_{+1}=\frac{1}{2}\left[\begin{array}{ll}
1 & 1 \\
1 & 1
\end{array}\right], \quad \mathbb{P}_{-1}=\frac{1}{2}\left[\begin{array}{cc}
1 & -1 \\
-1 & 1
\end{array}\right] \\
\mathbb{P}_{2}=\frac{1}{2}\left[\begin{array}{ll}
1 & 0 \\
0 & 1
\end{array}\right], \quad \mathbb{P}_{3}=\frac{1}{2}\left[\begin{array}{ll}
1 & 0 \\
0 & 1
\end{array}\right]
\end{gathered}
$$


Another interesting example is given by the mixture

$$
\mid \psi\}=p_{\alpha}^{1 / 2}[\alpha]|1\rangle+p_{\beta}^{1 / 2}[\beta]\left(c_{1}|1\rangle+c_{2}|2\rangle\right)
$$

of two pure states $\left|\psi_{\alpha}\right\rangle=|1\rangle$ and $\left|\psi_{\beta}\right\rangle=c_{1}|1\rangle+c_{2}|2\rangle$, where $c_{1}^{*} c_{1}+c_{2}^{*} c_{2}=1$ and $p_{\alpha}+p_{\beta}=1$. Note that arithmetically the same expression

$$
\mid \psi\}=\left(p_{\alpha}^{1 / 2}[\alpha]+p_{\beta}^{1 / 2} c_{1}[\beta]\right)|1\rangle+p_{\beta}^{1 / 2} c_{2}[\beta]|2\rangle
$$

would be more difficult to interpret. Hence, the random phases need to be factored out and the summation of different random phases, such as $p_{\alpha}^{1 / 2}[\alpha]+$ $p_{\beta}^{1 / 2}[\beta]$, is generally not permitted. If $c_{2} \rightarrow 0$ then $\left.\mid \psi\right\}$ becomes

$$
\mid \psi\}=\left(p_{\alpha}^{1 / 2}[\alpha]+p_{\beta}^{1 / 2}[\beta]\right)|1\rangle=|\psi\rangle
$$

which, as the mixture of the same pure states $\left|\psi_{\alpha}\right\rangle=\left|\psi_{\beta}\right\rangle=|1\rangle$, is conventionally interpreted as a pure state $|\psi\rangle$. If we follow this convention, then in this case the sum of the random phases can be formally interpreted as another random phase $[\gamma]$, that is $[\gamma]=p_{\alpha}^{1 / 2}[\alpha]+p_{\beta}^{1 / 2}[\beta]$.

In these work we distinguish pure and mixed states by different bra and ket symbols. Since, as canonical typicality indicates, the difference between pure and mixed states tends to be blurred for very large quantum systems, using the same bras and kets for both $|\psi\rangle$ and $\mid \psi\}$ may be more convenient. The random phases notation is a convenient notation for distinguishing pure and mixed states of quantum mechanics. In conventional quantum mechanics, the random phase multipliers $[\alpha],[\beta]$ are orthonormal and do not evolve in time. Spontaneous decoherence, however, corresponds to $[\alpha]$ being the same as $[\beta]$ initially but then evolving into stochastically independent quantities.

\section{References}

[1] W. H. Zurek, Environment-induced superselection rules, Phys. Rev. Lett. 26 (8) (1982) 1862-1888.

[2] S. Goldstein, J. L. Lebowitz, R. Tumulka, N. Zanghi, Canonical typicality, Phys. Rev. Lett. 96 (2006) 050403.

[3] S. Popescu, A. J. Short, A. Winter, Entanglement and the foundations of statistical mechanics, Nature Physics 2 (11) (2006) 754-758.

[4] N. Linden, S. Popescu, A. J. Short, A. Winter, Quantum mechanical evolution towards thermal equilibrium, Phys. Rev. E 79 (2009) 061103.

[5] V. Yukalov, Equilibration and thermalization in finite quantum systems, arXiv:1201.2781. 
[6] S. Abe, Maximum-power quantum-mechanical Carnot engine, Phys. Rev. E 83 (2011) 041117.

[7] J. McL. Emmerson, Symmetry principles in particle physics, Clarendon Press, Oxford, 1972.

[8] W. M. Gibson, B. R. Pollard., Symmetry principles in elementary particle physics, Cambridge Univ. Press, UK, 1976.

[9] G. Beretta, On the general equation of motion of quantum thermodynamics and the distinction between quantal and nonquantal uncertainties (MIT, 1981), arXiv: quant-ph/0509116 (2005).

[10] W. H. Zurek, Decoherence and the transition from quantum to classical revisited, Los Alamos Science (27) (2002) 1-26.

[11] A. Bassia, G. Ghirardi, Dynamical reduction models, Physics Reports. 379 (2003) 257-426.

[12] J. Ellis, J. S. Hagelin, D. V. Nanopoulos, M. Srednicki, Search for violations of quantum mechanics, Nuclear Physics, Section B 241 (2) (1984) 381-405.

[13] V. Weisskopf, E. Wigner, Berechnung der natiirlichen linienbreite auf grund der diracschen lichttheorie, Z. Phys. 63 (1930) 54-73.

[14] Y. K. Wang, I. C. Khoo, On the wigner-weisskopf approximation in quantum optics, Optics Communications 11 (4) (1974) 323-326.

[15] L. D. Landau, E. M. Lifshits, Theoretical Physics, Pergamon Press, Oxford ; Sydney, N.S.W, 1980.

[16] R. Penrose, Road to Reality: A Complete Guide to the Laws of the Universe, A. Knopf Inc., 2005.

[17] A. Y. Klimenko, U. Maas, CPT invariance and its implications for thermodynamics and kineticls, in: M. Pilotelli, G. P. Beretta (Eds.), Proc. of 12th Joint European Thermodynamics Conference, Brescia, Italy, 2013, pp. 294-303.

[18] Beringer J. et al. (Particle Data Group), The review of particle physics, Phys. Rev. D 86 (2012) 010001. 